\title{
Influence of Atmospheric Turbulence on the Performance of a High Dimensional Quantum Key Distribution System using Spatial Mode Encoding.
}

\author{
Brandon Rodenburg, ${ }^{1}$ Mehul Malik, ${ }^{1}$ Malcolm O'Sullivan, ${ }^{1}$ Mohammad Mirhosseini, ${ }^{1}$ \\ and Robert W. Boyd ${ }^{1,2}$ \\ ${ }^{1}$ The Institute of Optics, University of Rochester, Rochester, NY 14627 USA \\ ${ }^{2}$ Department of Physics, University of Ottawa, Ottawa ON K1N 6N5 Canada \\ Brandon.Rodenburg@gmail.com
}

\begin{abstract}
The effects of atmospheric turbulence on a the channel capacity of a free-space quantum key distribution system with information encoded on the transverse modes of the photon are studied theoretically and experimentally.
\end{abstract}

(C) 2011 Optical Society of America

OCIS codes: (010.1330) Atmospheric turbulence; (270.5585) Quantum information and processing; (270.5565)

Quantum communications; (270.5568) Quantum cryptography

There has been a great deal of interest in exploring the possibility of a quantum communication channel in which information is impressed on the spatial degrees of freedom of a single photon light field [1,2] for a variety of applications in communications and in particular for quantum key distribution (QKD) [3]. As the spatial modes of light of a free-space channel form an infinite-dimensional Hilbert space, the amount of information that a single photon can carry is in principle infinite, allowing for a clear pathway to scale the transmission rates. In addition a larger state space can be exploited to obtain an increase in security for a QKD system $[4,5]$.

The orbital angular momentum (OAM) basis set has been singled out as a promising candidate for a spatial encoding scheme [6] as it is easily synthesized in the laboratory, has well-studied quantum optical properties, and can be efficiently sorted [7]. In addition, the mutually unbiased basis (MUB) states that are required for implementing the BB84 QKD protocol [8] are simply spatially localized angular slices which we call the angular modes (ANG). The form of these two sets of modes is given by:

$$
\Phi_{l}^{O A M} \sim e^{i l \phi}, \quad \Phi_{n}^{A N G} \sim \sum_{l=-L}^{L} \Phi_{l}^{O A M} e^{i 2 \pi n l /(2 L+1)}
$$
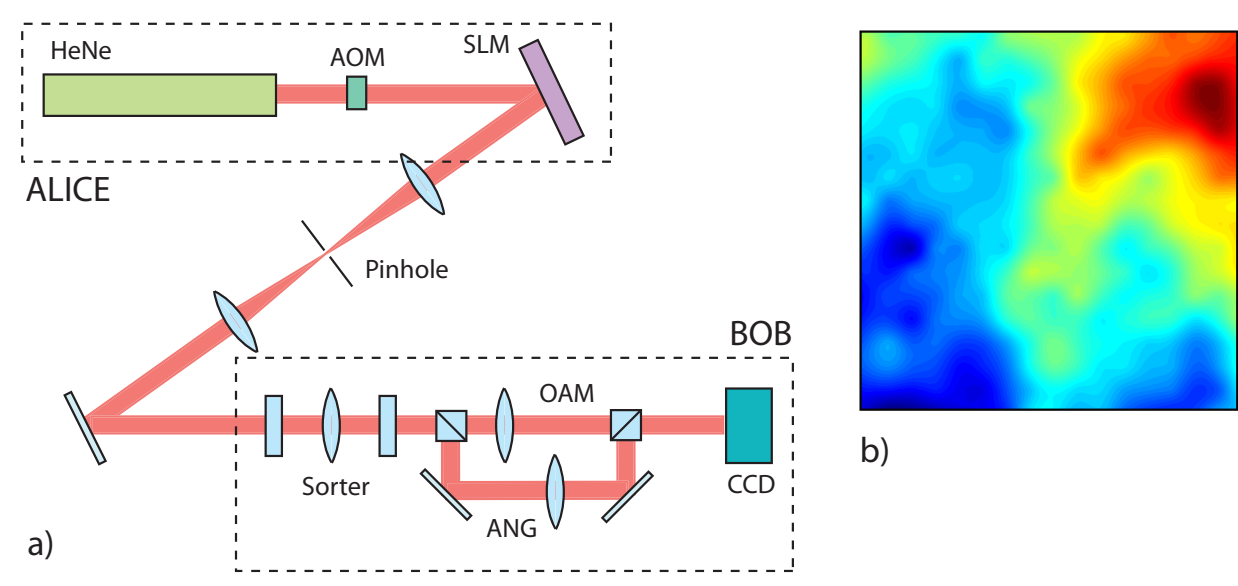

b)

Fig. 1. a) Experimental realization of a high dimensional QKD system using OAM and ANG modes. b) Sample Kolmogorov turbulence phase screen that is added to the input modes on the SLM. 
As is potentially the case for any free-space channel, turbulence effects in the communication arm between the sender and receiver can lead to a decrease in system performance. In order to implement such communication systems, these effects need to be characterized and well understood. Turbulence that is not too strong can be modeled by mapping the turbulence onto a phase function in the entrance pupil of the receiver. This phase function is described by standard Kolmogorov theory in terms of a specific form of the correlation statistics characterized by the length scale parameter, $r_{0}$, often referred to as "Fried's parameter" [9].

Numerical studies on the effects of such a thin phase turbulence on OAM based quantum communication systems have been developed in recent years $[10,11]$. We experimentally realize such modeling as shown in Fig. 1. Our source is an attenuated HeNe laser with a pulse train carved out by an acousto-optic modulator (AOM). Full spatial phase and amplitude modulation of the field is achieved with a phase only spatial light modulator (SLM) and 4f system [12]. Random Kolmogorov turbulence phase is added to the input states generated by Alice on the SLM (Fig. 1). These states are then transmitted over a free-space channel and received by Bob, who sorted them using the device in [7]. The output is then spatially binned on a CCD for characterization (the CCD is replaced by a fiber array connected to single photon APDs when the system is operating as a QKD system).
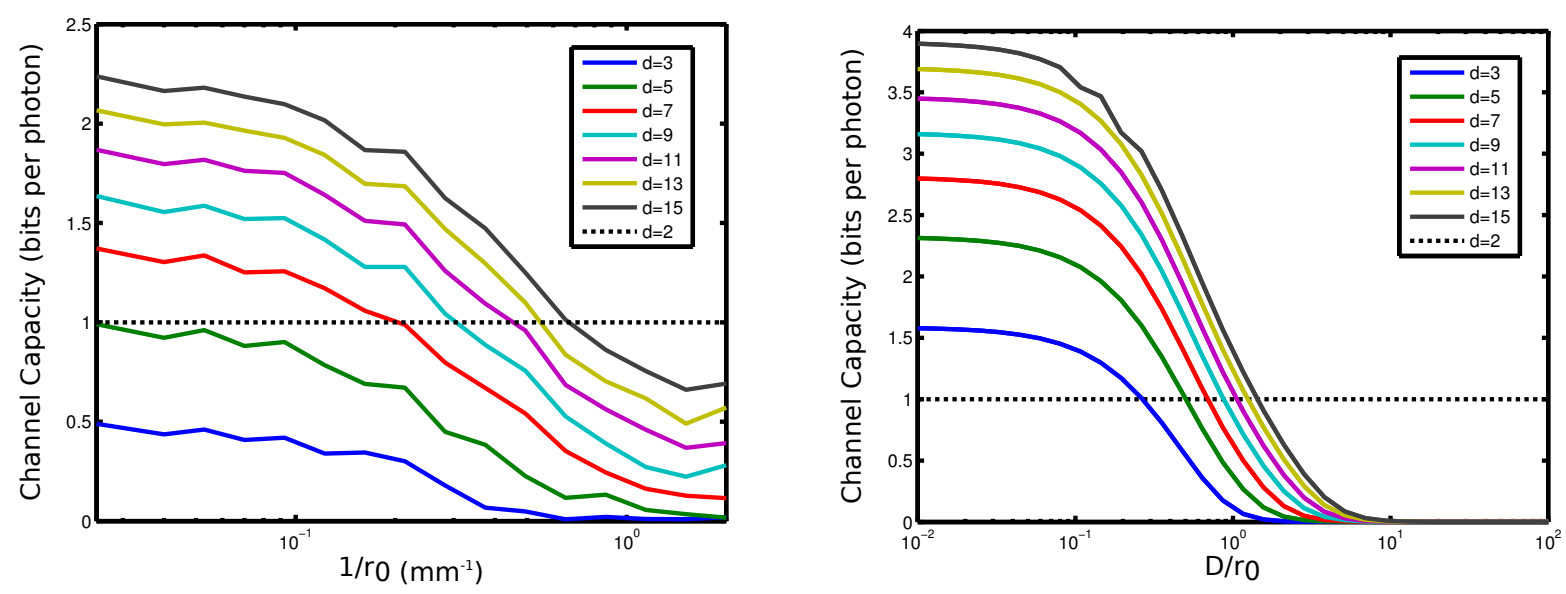

Fig. 2. Channel capacity as a function of the turbulence strength for a $d$-dimensional state space. Left: Preliminary experimental data. Right: Numerically calculated data for a perfect system affected by turbulence. $D$ is the diameter of the receiver's entrance pupil.

Preliminary results of our experimental studies are shown in Fig. 2. The measured channel capacity of the system as a function of the turbulence parameter $r_{0}$ for state space size, $d$, is plotted in Fig. 2 . The dotted line represents the case of a system encoding 1 bit per photon (i.e. for a 2 dimensional state-space) for comparison. In addition, the numerically calculated values of the channel capacity for a system in which turbulence is the only source of error is plotted. As expected, turbulence diminishes the performance of the system, and modes with size of order $r_{0}$ perform only as well as the minimum dimesional state space of $d=2$. These channel capacities apply to the OAM states only, as the angular modes are spatially localized modes and are thus unaffected by turbulence in the thin-phase limit. Currently we are expanding our methods to include the effects of turbulence even in the thick turbulence limit.

\section{References}

1. M. Bourennane, A. Karlsson, and G. Björk, "Quantum key distribution using multilevel encoding," Phys. Rev. A 64, 012,306 (2001).

2. A. Mair, A. Vaziri, G. Weihs, and A. Zeilinger, "Entanglement of the orbital angular momentum states of photons." Nature 412, 313-6 (2001).

3. N. Gisin and R. Thew, "Quantum communication," Nature Photonics 1, 165-171 (2007).

4. M. Bourennane, A. Karlsson, G. Bj rk, N. Gisin, and N. J. Cerf, "Quantum key distribution using multilevel encoding: security analysis," Journal of Physics A: Mathematical and General 35, 10,065-10,076 (2002).

5. N. Cerf, M. Bourennane, A. Karlsson, and N. Gisin, "Security of Quantum Key Distribution Using d-Level Systems,” Phys. Rev. Lett. 88 (2002). 
6. G. Gibson, J. Courtial, M. J. Padgett, M. Vasnetsov, V. Pas'ko, S. M. Barnett, and S. Franke-Arnold, "Free-space information transfer using light beams carrying orbital angular momentum," Opt. Express 12, 5448 (2004).

7. G. Berkhout, M. Lavery, J. Courtial, M. Beijersbergen, and M. Padgett, "Efficient Sorting of Orbital Angular Momentum States of Light,” Phys. Rev. Lett. 105 (2010).

8. C. H. Bennett and G. Brassard, "Quantum cryptography: Public key distribution and coin tossing," in "Proceedings of IEEE International Conference on Computers Systems and Signal Processing,", vol. 175 (Bangalore, India, 1984), vol. 175, pp. 175-179.

9. D. L. Fried, "Statistics of a Geometric Representation of Wavefront Distortion," J. Opt. Soc. Am. 55, 1427 (1965).

10. G. A. Tyler and R. W. Boyd, "Influence of atmospheric turbulence on the propagation of quantum states of light carrying orbital angular momentum," Opt. Lett. 34, 142 (2009).

11. C. Paterson, "Atmospheric Turbulence and Orbital Angular Momentum of Single Photons for Optical Communication," Phys. Rev. Lett. 94 (2005).

12. M. T. Gruneisen, W. A. Miller, R. C. Dymale, and A. M. Sweiti, "Holographic generation of complex fields with spatial light modulators: application to quantum key distribution," Appl. Opt. 47, A32 (2008). 